\title{
Economic Explanation, Ordinality and the Adequacy of Analytic Specification
}

by

Donald W. Katzner

Professor of Economics

Department of Economics

University of Massachusetts\Amherst

Amherst, MA 01003

USA

Email: dkatzner@econs.umass.edu
Peter Skott

Professor of Economics

Department of Economics

University of Massachusetts \Amherst Amherst, MA 01003

USA

Email:pskott@econs.umass.edu

Revised: 19 February, 2004

\begin{abstract}
:
This paper examines the implicit links between models containing ordinal variables and their underlying unquantified counterparts that are necessary to make the former viable theoretical constructions. It is argued that when the underlying unquantified structure is unknown, the permissible transformations of scale applicable to the ordinal variables have to be restricted beyond that which is permitted by dint of the ordinality itself. The possibility of an underlying structure being known but unspecified is also considered. In the case of the efficiency wage model, the only usable transformations of the ordinal effort scale are those which are multiples of each other.
\end{abstract}

Key Words: Ordinal variable, unquantified variable, effort, efficiency wage theory.

JEL classification: B41 


\section{Economic Explanation, Ordinality, and the Adequacy of Analytic Specification ${ }^{1}$}

Analytical economics holds as its objective and province of argument the explanation of economic behavior, decision, and choice. Analysis within its purview may or may not assume predictive competence as distinct from explanation; and it may or may not be readily adaptable to detailed empirical description and corroboration. The methods employed can, among other things, be directed to questions of agent optimization and welfare, or to those that bear on wider issues of aggregative activity or social benefit. In analyses of this sort, economists generally and typically construct models by postulating relational structures that have specific properties. Those contemplated relations, which may or may not be well specified and written in (ordinally, cardinally, or ratio ${ }^{2}$ ) quantified functional form, are often reflective of deeper, or onestage-removed, determinant forces. But in any case, it is well known that a number of difficulties stand in the way of such an approach.

First, it is obvious that for an analysis based on relational structures to have sustainable meaning, the structures and their properties must remain fixed throughout the investigation and over all relevant, analytically manipulative procedures. But second, questions of analytical legitimacy arise when one or more of the variables incorporated in the argument are only ordinally measured while all remaining variables are taken to be at least cardinally quantified. In such a case, it is possible that the structures and properties assumed, if expressed only in the numerical terms of the quantified variables (ordinal, cardinal, and ratio), are dependent to a considerable extent on the scales on which the ordinal variables are measured. It follows that in the presence of such ordinality, the properties can often be altered or entirely lost upon application of certain increasing transformations of scale with respect to the ordinal variables. Thus, for example, the structure $h(y)=-y^{2}$, where $-\infty \leq y \leq \infty$ and $y$ is only ordinally measured, has a unique maximum at $y=0$. But with $\lambda>1$ a fixed number, applying the increasing transformation $T(y)=-\lambda^{-y}$ to $y$ and replacing $y$ in $h$ 
by the ordinally equivalent transformed variable $T(y)$, results in the structure $h(T(y))=-\lambda^{-2 y}$ which has no maximum anywhere.

The problem of the preservation of structures and their properties across transformations of scale does not arise in certain well-known cases, like that of the utility function in the derivation of consumer demand functions, where ordinality appears with respect to a dependent variable. Nor does it usually arise when cardinal or ratio scales are involved because, generally speaking, the transformations permitted in those circumstances are insufficient to cause significant alteration or loss of information pertaining to the postulated structures and their properties. In the present paper, then, only scale changes with respect to ordinal scales are considered. To the extent that variables measured on cardinal and ratio scales are introduced, those scales are assumed fixed and no modification in them is permitted. With regard to dependent variables, only those situations in which scale changes have the potential to cause difficulties are discussed.

More specifically, the paper begins with a general discussion of ordinality in reference to a single structural relation. Three approaches to handling that ordinality are identified: The first (Approach A) is to begin with the relation in an unquantified state and then employ specific ordinal scales to translate it into numerical form. This procedure, which introduces at the start of the analysis the theoretical challenge of according meaning and manipulability to nonquantified variables according to the rules of "analysis without measurement, ${ }^{3}$ ensures that the original structure and its properties do not modify with ordinal scale changes. Indeed, they are independent of all ordinal measurement scales. Approach A is illustrated in Katzner’s (1979: Chs. 5,6) implicit construction of numerical representations of individual "quality of life technologies” relating “career-living situations” to "qualities of life.” The two alternative approaches are, first, to postulate the existence of a numerical function that reflects a known but unspecified underlying, unquantified structure (Approach B) and, second, to assume that that underlying structure is not only unspecified, but also unknown (Approach C). Both of these approaches, as will be argued, have serious weaknesses. 
There are many studies in the Economics literature that, since their authors ignore underlying structures and do not specify how their questionably quantified variables are to be measured, can be taken to exemplify either Approach B or C. To name three (more will be identified momentarily), Akerlof includes variables representing the "quality” of an automobile (1970: p. 490) and a person’s “reputation” (1980: p. 754) in quantified mathematical equations, and Becker and Lewis (1973) derive the constrained maximization of a utility function with respect to "child quality” and other variables.

After a general description and comparison of the three approaches, Approach $\mathrm{C}$ and one of its limitations is considered in greater detail. The paper concludes by examining the special case in which the dependent variable of the single structural relation (with one independent, ordinally measured variable) is assumed to be measured on a ratio scale. This discussion is taken up in reference to all three Approaches A, $\mathrm{B}$, and $\mathrm{C}$.

The range of difficulties suggested in the foregoing have presented economists with problems that have seemed analytically intractable and have raised uncertainty regarding the use and manipulability of variables measured on differently specified scales (ordinal, cardinal, and ratio), quite apart from the knotty problem of nonquantifiabity. ${ }^{4}$ Such problems can be exemplified by reference to certain efficiency wage and other models. There the issues involved have led to controversy over the viability of the internal logic of the particular models that have been employed. For example, Currie and Steedman (1993) and Katzner (1998) have correctly argued (in part) that, taken on face value, efficiency wage models as presented in the Economics literature (e.g., Stiglitz (1987: 27, n. 47) are unsustainable: Effort can only be ordinally measured, and the use in those models of some arbitrary ordinal representation of effort as an argument of the production function leaves that production function inadequately specified. Skott (1997a), on the other hand, argues, also correctly, that introducing an implicit link between unquantified effort and (quantified) output pins down the quantified production function (relating ordinally measured effort to output) sufficiently to eliminate the problem. More specifically, the stipulated production function in efficiency wage models is conditional on the particular choice of the ordinal representation of effort, and any change in that choice will necessitate a corresponding modification in the production function. (A similar assertion is 
latent in Leslie (1995).) However, Skott provides no details concerning the relation of that link between unquantified effort and output to the quantified production function, although his suggested procedure will be seen in what follows to be an example of Approach B. An associated controversy has arisen between Leslie (1995) and Skott (1997b) with the latter taking a position similar to his stance on the efficiency wage case in relation to ordinally measured "education" in a model of human capital theory. ${ }^{5}$ One of the purposes of this paper is to make explicit Skott s implicit Approach B links between such variables as unquantified effort and output, and to examine their relation to the functions, like the quantified production function, to which they correspond. Implications are drawn not only for efficiency wage models, but also for a wider class of models in general. It will emerge that analyses that fall in the category of Approach B, like those of Approach C, carry the potential of previously unsuspected and damaging problems.

\section{The Three Approaches}

\section{I.A Approach A -- Explicit Links}

To formally characterize what has been referred to as Approach A, focus attention on functions of a single variable (see Katzner (1983: 53-58)). At the outset, let $e$ and $x$ denote unquantified or unmeasured variables ${ }^{6}$ defined by the values or objects over which they are permitted to vary. Were $e$, say, to represent effort, the objects over which $e$ varies might be verbal descriptions, each depicting a manifestation of effort thought to be relevant to the issue at hand. Denote the collections of objects that $e$ and $x$ may assume as values by, respectively, $E$ and $X$. Take $f$ to be a function associating to each value of $e$ in $E$ a unique value of $x$ in $X$, and write

$$
x=f(e) .
$$

Suppose now that $e$ and $x$ are capable of ordinal measurement only. Then, with respect to $e$, say, the objects of $E$ are ordered according to some property such as longness, hotness, or pleasure, and that ordering, call it 
$\succcurlyeq_{e}$, is fully represented on a numerical scale that is unique up to increasing (and continuous)

transformations. Moreover, the ordering $\succcurlyeq_{e}$ is reflexive, transitive, and total on $E$. Similar statements may be made in relation to $x$ where, in this case, the underlying ordering is designated by $\succcurlyeq_{x}$. Denote the measured values of $e$ and $x$ on the particular ordinal scales in use by, respectively, $\varepsilon$ and $\chi$. Then with (1) specified, a numerical function relating $\varepsilon$ and $\chi$, namely

$$
\chi=F(\varepsilon)
$$

is implied. ${ }^{7}$ Clearly $F$ depends on the choice of scales on which $e$ and $x$ are measured. As long as (1) is to be maintained as an assumed structural relation, any alteration in one or both scales, unless, in the latter case, the two alterations exactly cancel each other out, necessarily modifies $F$.

Suppose now that increasing transformations $T$ and $S$ are applied to the scales on which, respectively, $e$ and $x$ are measured. Denote the new variables representing $e$ and $x$ by, respectively, $\varphi$ and $\xi$ so that

$$
\varphi=T(\varepsilon) \text { and } \quad \xi=S(\chi)
$$

Then, as indicated above, $F$ has to be altered to maintain (1). Write the new representation of (1) as

$$
\xi=G(\varphi)
$$

It is easy to see that, as long as appropriate inverses exist ( $S$ and $T$ already have inverses since they are increasing), any one of $F, G, S$, and $T$ can be secured from the remaining three. For example, substituting (4) into the right-hand equation of (3), and using (2), 


$$
G(\varphi)=S(F(\varepsilon))
$$

If $G$ has an inverse, $G^{-1}$, then

$$
\varphi=G^{-1}(S(F(\varepsilon)))
$$

so that $T$ may be viewed as the composition of $F, S$, and $G^{-1}$. That is,

$$
T=G^{-1} \circ S \circ F,
$$

and specification of $F, G$, and $S$ determines $T$. Clearly, since $S$ and $T$ are increasing, implicit restrictions are imposed on $F$ and $G$ in this formulation. Alternatively, combining the left-hand equation of (3) with (4) and (2) gives, if $F$ has an inverse,

$$
\xi=G\left(T\left(F^{-1}(\chi)\right)\right)
$$

whence

$$
S=G \circ T \circ F^{-1}
$$

Similarly, $F=S^{-1} \circ G \circ T$ and $G=S \circ F \circ T^{-1}$.

Economists seem to prefer to deal with functions in numerical form rather than those in their underlying, unquantified state. Moreover, the preceding analysis suggests that functions like $F$ ought to be constructed by specifying $f$ and numerical measures of the values of $e$ and $x{ }^{8}$ But this is not easy to do for at 
least two reasons. First, economists are neither familiar with nor comfortable dealing with the unquantified. Second, many of the techniques that they employ in their analyses render it necessary that the numerical functions under consideration be continuous or differentiable. Continuity and differentiability as invoked by economists, however, usually require functions with nondenumerably infinite numbers of elements in their domains and ranges. And, although it is possible to conceive of unquantified functions with nondenumerably infinite domains and ranges, ${ }^{9}$ because unquantified functions can be specified only by explicitly indicating which dependent variable values are identified with which independent variable values, the finiteness of our world does not permit the specification of more than a finite number of relational points under $f$. Moreover, the arithmetic operation of subtraction needed to calculate a derivative as the limit of the slopes of straight-line segments is not available because numbers are not present. Thus to employ the approach to $F$ described above, a natural extension of Katzner (1998: 20-21) and already referred to as Approach A, means that economists would have to give up analytical techniques that have been quite useful and fruitful when all variables are measured on at least cardinal scales.

There are, however, two alternative, if somewhat less satisfying, approaches.

\section{I.B Approach B -- Unspecified Links}

What has been earlier identified as Approach B is to assume that there is an underlying function of the form of (1) that is known but unspecified (that is, the function is assumed to exist but its form is not articulated), that there are also known but unspecified ordinal scales on which $e$ and $x$ are measured, and that together they give rise to a function $F$ in (2) that has certain known and fully specified properties. From the perspective of Approach B, then, analysis begins by postulating the existence of a function $F$ that represents $f$ and that possesses certain numerical properties which may possibly include continuity or differentiability.

But unlike Approach A, the process of specifying $f$ and translating it into $F$ is suppressed. Clearly the properties of $F$ are assumed to mirror those of $f$, and if the full force of the properties of $F$ are to be maintained, then any changes in scale mean that $F$ must be adjusted to preserve $f$ as in (4). Of course the previous mathematics relating $F, G, T$, and $S$ remains intact. (Were continuity or differentiability assumed 
of $F$, then $f$ must be thought of as having a nondenumerably infinite domain and range.) This is the approach taken by Skott (1997a, 1997b) mentioned earlier. In effect, the approach surmounts the problem of nonquantifiability by assuming it away and by resting its argument on assumed, even though unspecified, ordinality.

Approach B is less satisfactory than Approach A for three reasons. First it requires greater assumption content than approach A. In effect, both (1) and (2) must be assumed instead of having the latter derived from the former. Second, it is necessary to assume that certain things are known to exist without being able to specify what they are. Third (this arises in part from the second reason), the precise relationship between the ordinal numbers and the variable values they represent cannot be stated, and exactly what is being assumed about $f$ and its properties is not clear. In short, a significant portion of the relationship to reality of the assumptions imposed in the model is hidden by the veil of ordinal numbers with respect to which those assumptions are expressed.

\section{I.C Approach C -- Unknown Links}

The third approach, previously called Approach C, is characterized as the same as Approach B except that both $f$ and the ordinal scales on which $e$ and $x$ are measured are taken to be not only unspecified, but also unknown. The analytical content of this approach, however, raises several conceptual issues that warrant consideration before proceeding. First, it may be vaguely surmised or assumed in the explanation of certain economic conditions that an underlying, unquantified relationship exists and determines a particular outcome. (For example, a firm's reputation may influence the demand for its output.) But secondly, it may also be reckoned in such a case that there is no way in which ordinal scales can be meaningfully employed to numerically quantify the strength of the forces inherent in that relationship. (In the previous example, how might the quality of the firm's reputation be measured?) Thirdly, while the general nature of those forces is thereby recognized, residual uncertainty and lack of clarity exist as to the manner in which those forces transmit their impacts to the outcome that is in view. (With respect to the example, in what precise 
way does the quality of the firm's reputation contribute to the demand for its output?) This, then, is one illustration of the circumstances in which Approach C might be brought into play.

It should be noted that Approach $\mathrm{C}$ does not suffer from the deficiencies associated with the second reason given above why Approach B is less satisfactory than Approach A. But it is still less satisfactory than A for reasons one and three that were stated there. Moreover, Approach C is subject to a certain kind of arbitrariness that will be explored in Section II below.

\section{I.D Comparing the Three Approaches}

To further illuminate what is involved, it is worth summarizing and setting against each other the distinctives of the three approaches that have now been described. In Approach A it is assumed that the relevant qualitative forces underlying and contributing to the statement of $F$ in (2) as derived from the “ordinalization” of (1) are, for the purposes in hand, known and corralled with respect to the specific ordinal scales introduced. In Approach B the possibility is contemplated that the underlying forces and their relation to ordinal scales, though still present, need not be expressed, and that analysis could begin by directly positing $F$ in (2). With regard to Approach C, however, a much higher degree of relative ignorance is assumed. Not only are the underlying forces both vague and unfettered by ordinally specified scales, but now it is reckoned that no such underlying ordinal relations that might pin them down can be known.

An alternative statement of Approach $\mathrm{C}$ and its relation to Approaches A and B is as follows: In both Approaches $\mathrm{A}$ and $\mathrm{B}$ the relation $f$ is in one way or another the starting point of the argument. That is the case in Approach B because $F$ is anchored to (the unspecified) $f$ using the given (though unspecified) ordinal scales on which $e$ and $x$ are measured. But, as has been explained, that $f$ and those scales are suppressed and the assumption is made that it is not necessary to introduce them into the analysis. In Approach C, on the other hand, $F$ serves as its own anchor without any grounding in an underlying relation such as $f$ and, as a result, the mathematics described earlier that relates $F, G, T$, and $S$ is rendered irrelevant. Moreover, for $F$ to remain a meaningful anchor as the scales on which the ordinal variables are measured modify (that is, to ensure the analytically relevant properties of $F$ remain unchanged), the transformations of 
scale permitted have, as will be shown, to be restricted. Approach $\mathrm{C}$ is the focus of attention in the next section. But first it is worth presenting an example to illustrate the issues and differences involved in the three Approaches A, B, and C.

Let the variables $e$ and $x$ denote, respectively, unquantified effort and unquantified pleasure. Suppose there are two values of effort, $e^{\prime}$ and $e^{\prime \prime}$, and two values of pleasure, $x^{\prime}$ and $x "$. Introduce

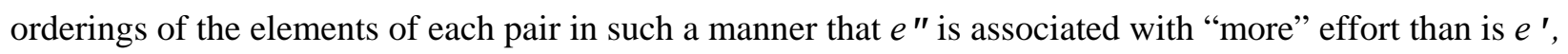
and $x$ " is associated with "more" pleasure than is $x^{\prime}$. Assume $f$ maps $e^{\prime} \rightarrow x^{\prime}$ and $e^{\prime \prime} \rightarrow x^{\prime \prime}$. Then under $f$, more effort corresponds to more pleasure. The latter characteristic of $f$, often referred to as order preservation, is the only structural property considered in this example.

Now let $e$ and $x$ be measured on independent ordinal scales that preserve the ordering relations among, respectively, the values of $e$ and $x$. Suppose the measured values are $e^{\prime} \rightarrow \varepsilon^{\prime}=2, e^{\prime \prime} \rightarrow \varepsilon^{\prime \prime}=3$, $x^{\prime} \rightarrow \chi^{\prime}=4$, and $x^{\prime \prime} \rightarrow \chi^{\prime \prime}=9$. Then more effort and more pleasure are identified with higher numbers. (In the usual parlance, the values of $\chi$ are called utility values.) Furthermore, according to (2), $f$ expressed in numerical form becomes, in this example,

$$
\chi=F(\varepsilon)=\varepsilon^{2}
$$

on the two-element domain $\varepsilon=\varepsilon^{\prime}$ and $\varepsilon=\varepsilon^{\prime \prime}$. Clearly, $F(2)=4$ and $F(3)=9$. The order-preservation property of $f$ is reflected in $F$ : greater effort (a higher value of $\varepsilon$ ) is associated with greater pleasure or utility (a higher value of $\chi$ ). It is evident that (7) can only preserve this underlying ordering relation when the domain of $F$ is restricted to nonnegative numbers. Any increasing transformation that would shift the domain to negative numbers will not leave that ordering relation unchanged. From the perspective of Approach A, however, the way to ensure that, after such changes in scales are introduced, $F$ still represents $f$ and maintains its property of order preservation is to modify $F$ so as to offset the scale modifications. If, say, the increasing transformation 


$$
\varphi=T(\varepsilon)=-2^{-\varepsilon},
$$

where $\varepsilon>0$, were applied to the $\varepsilon$-scale so that $\varphi^{\prime}=T(2)=-1 / 4$ and $\varphi^{\prime \prime}=T(3)=-1 / 8$, and if $S$ were the identity transformation (a special case of an increasing transformation), then solving (8) for $\varepsilon$ and substituting the result into (7) gives the following expression for (4):

$$
\xi=G(\varphi)=\left[\frac{\ln (-1 / \varphi)}{\ln 2}\right]^{2}
$$

where, like (7), $G(-1 / 4)=4$ and $G(-1 / 8)=9$. Clearly, the measured values of $e^{\prime}$ and $e^{\prime \prime}$ have become $e^{\prime} \rightarrow$ $\varphi^{\prime}=-1 / 4$ and $e^{\prime \prime} \rightarrow \varphi^{\prime \prime}=-1 / 8$, and $G$ preserves the same order as $f$ since greater effort (now a larger value of $\varphi$ ) still corresponds to greater pleasure or utility (a larger value of $\xi=\chi$ ). Thus an appropriate modification of $F$ has been made in response to the change in scale on which $e$ is measured. A similar argument without the explicit link to $e^{\prime}, e^{\prime \prime}, x^{\prime}, x^{\prime \prime}$, and $f$ applies in the case of Approach B.

However, if, as in Approach C, $F$ serves as its own anchor in the sense previously explained (recall $f$ is unknown), then, at least in so far as the ordinality of the measured variables is concerned, there is in that case no difference between (7) as above and (7) with $\varepsilon$ replaced by the transformed variable $-2^{-\varepsilon}$. That is, (7) and

$$
\chi=F(T(\varepsilon))=\left(-2^{-\varepsilon}\right)^{2}
$$

are equivalent formulations of the same relation in that they contain the same information. But with respect to (9), $F(T(2))=1 / 16$ and $F(T(3))=1 / 64$. Thus $F \circ T$ reverses order: greater effort as represented in a larger 
value of $\varepsilon$ corresponds to less pleasure or utility as indicated by a smaller value of $\chi$. Therefore, since $F$ is the anchor and since its relevant property (order preservation) has to be preserved, application of the increasing transformation $T(\varepsilon)=-2^{-\varepsilon}$, although perfectly legitimate in terms of the ordinality of the scales involved, has to be ruled out under the methodology of Approach C. This illustrates a fundamental fact that ought to be kept in mind: When $F$ serves as the analytical anchor, certain scale transformations of the variables involved have to be excluded if the properties of $F$ are to be maintained as components of the structural foundation of the analysis. Part of the next section generalizes the argument and makes it more precise.

\section{Scale Transformations under Approach C}

When starting out to do an analysis, there are two basic steps that are relevant to present considerations --

first identify the variables to be accounted for, and second construct or assume relations among them. If any of the variables are ordinal variables, problems relating to that ordinality may arise at both levels.

In the process of identifying the ordinal variables, the scales on which they are measured have to be, at least implicitly, fixed. That is, what version of the numbers should be used? Should they be those that present themselves by some means to the investigation, or should they be transformed in one way or another? Suppose these questions are answered first. Now let the hypothesized relations of the analysis be introduced. In the case of Approach B, these relations are reflections of known (but unspecified) underlying forces, and changes in ordinal scales cannot be permitted to destroy the representation of those forces in the hypothesized relations. In this case, then, the relations have to modify as in (4) with any scale changes.

Now assume, from the perspective of Approach C, that the hypothesized relations are thought to mirror unknown underlying forces. These relations and their analytically significant properties have to be kept intact because they are the only representation of those forces in the analysis. But it is still reasonable and appropriate to ask how changes in the initial choice of scales would affect the hypothesized relations, and, as described at the end of the preceding section, to rule out those scale changes that interfere with and 
modify the analytically significant properties. In the context of equation (2), say, before such interference is taken into account, all functions of the form

$$
\chi=S^{-1}(F(T(\varepsilon)))
$$

where $T(\varepsilon)$ and $S(\chi)$ are substituted in place of, respectively, $\varepsilon$ and $\chi$ in (2), and $S$ and $T$ are the increasing transformations of (3), are potentially equivalent starting points for the analysis. Observe that (9) is a special case of (10). However, as previously indicated, the only increasing scale transformations that can be permitted are those that preserve the analytically significant properties, and hence the only functions $S^{-1} \circ F \circ T$ alternative to $F$ that are pertinent to present discussion are those that exhibit them. ${ }^{10}$ Were increasing transformations applied that did not preserve those properties, then the relevant properties associated with $S^{-1} \circ F \circ T$ would deviate from those of $F$. Such transformations, therefore, would have to be discarded. But ordinality by itself means that no information in the ordinally measured variables is lost by administering increasing transformations of scale to them, and hence, from this perspective, that all increasing transformations should be permissible. It follows that the more scales that have to be excluded to preserve the analytically relevant properties of $F$ when that ordinality is imported to purported explanatory relations, the greater the arbitrariness introduced into the analysis. That arbitrariness, which, as suggested earlier, is inherent in Approach C, is a serious drawback in the construction of economic explanation.

The problem of arbitrariness arising from the necessity to discard legitimate transformations of scale in Approach C has already been illustrated in the example of Section I with respect to order preservation for functions of a single variable. That argument, although easily generalized, is not developed further here.

Similar considerations apply, and again point to the limitations of Approach C, to the circumstance in which $F$ has a unique maximum (Katzner (1983: 107)). For in the absence of any restrictions imposed by the necessity of preserving the properties of $F$, all increasing transformations of scale are permissible, and thus the possibility cannot be ruled out that maxima of $F$ intended to be reflective of unknown underlying 
forces might actually not exist under some transformations of scale. An example has previously been provided in the introduction to this paper.

Matters are further complicated when $f$ is a function of more than one variable since, in that case, at least one additional issue arises. To provide a simple illustration, suppose there are only two independent variables. Let $F$ be the Approach-C anchor of an analysis where

$$
\chi=F\left(\varepsilon_{1}, \varepsilon_{2}\right)
$$

on a suitable domain, and where $\varepsilon_{1}$ and $\varepsilon_{2}$ are ordinally measured variables. Then $F$ induces an ordering relation $\succ$ on its domain in the usual way:

$$
\left(\varepsilon_{1}^{\prime}, \varepsilon_{2}^{\prime}\right) \succ\left(\varepsilon_{1}^{\prime \prime}, \varepsilon_{2}^{\prime \prime}\right) \quad \text { if and only if } F\left(\varepsilon_{1}^{\prime}, \varepsilon_{2}^{\prime}\right)>F\left(\varepsilon_{1}^{\prime \prime}, \varepsilon_{2}^{\prime \prime}\right)
$$

In general, the ordering $\succ$ generated by $F$ is independent of those that define the ordinality of $\varepsilon_{1}$ and $\varepsilon_{2}$. Now, in accordance with Approach C, replace $\varepsilon_{1}$ and $\varepsilon_{2}$ in (11) by $\varphi_{1}$ and $\varphi_{2}$, where

$$
\varphi_{1}=T^{l}\left(\varepsilon_{1}\right) \text { and } \varphi_{2}=T^{2}\left(\varepsilon_{2}\right) \text {, }
$$

and $T^{1}$ and $T^{2}$ are arbitrary, increasing transformations. Then $F$ becomes

$$
\chi=F\left(\varphi_{1}, \varphi_{2}\right)
$$

on an appropriately altered domain. Substituting (13) into (14), the original $F$ is modified to $F \circ\left[T^{1}, T^{2}\right]$, that is, 


$$
\chi=\left(F \circ\left[T^{1}, T^{2}\right]\right)\left(\varepsilon_{1}, \varepsilon_{2}\right)=F\left(T^{1}\left(\varepsilon_{1}\right), T^{2}\left(\varepsilon_{2}\right)\right),
$$

and $F \circ\left[T^{1}, T^{2}\right]$ induces a second ordering relation on the original domain of $F$ as in (12). Clearly, these ordering relations need not be the same. To illustrate that possibility, consider the function $F\left(\varepsilon_{1}, \varepsilon_{2}\right)=\varepsilon_{1}$ $+\varepsilon_{2}$ and the vectors $\left(\varepsilon_{1}{ }^{\prime}, \varepsilon_{2}{ }^{\prime}\right)=(2,0)$ and $\left(\varepsilon_{1}{ }^{\prime \prime}, \varepsilon_{2}{ }^{\prime \prime}\right)=(0,1)$. Then $F\left(\varepsilon_{1}{ }^{\prime}, \varepsilon_{2}{ }^{\prime}\right)>F\left(\varepsilon_{1}{ }^{\prime \prime}, \varepsilon_{2}{ }^{\prime \prime}\right)$ and $\left(\varepsilon_{1}{ }^{\prime}, \varepsilon_{2}{ }^{\prime}\right) \succ$ $\left(\varepsilon_{1}^{\prime \prime}, \varepsilon_{2}{ }^{\prime \prime}\right)$. But with $T^{1}\left(\varepsilon_{1}\right)=\varepsilon_{1}$ and $T^{2}\left(\varepsilon_{2}\right)=3 \varepsilon_{2}$, the ordering of $\left(\varepsilon_{1}{ }^{\prime}, \varepsilon_{2}{ }^{\prime}\right)$ and $\left(\varepsilon_{1}{ }^{\prime \prime}, \varepsilon_{2}{ }^{\prime \prime}\right)$ under the original $\succ$ is reversed since $F\left(T^{l}\left(\varepsilon_{1}^{\prime}\right), T^{2}\left(\varepsilon_{2}^{\prime}\right)\right)<F\left(T^{1}\left(\varepsilon_{1}^{\prime \prime}\right), T^{2}\left(\varepsilon_{2}^{\prime \prime}\right)\right)$. Returning to the general situation, if the original ordering induced by $F$ is to be preserved as an analytically significant property, then all transformations $T^{1}$ and $T^{2}$ that modify that ordering have to be excluded. Thus possible instances of the kind of arbitrariness that damaged the analytical viability of Approach $\mathrm{C}$ with respect to functions of a single variable can also arise for functions of more than one variable, even if $F$ is increasing everywhere in both of its arguments.

Similar arbitrariness extends to attempts to preserve the solutions of systems of simultaneous equations involving ordinal variables under increasing transformations of scale. Except for the following illustration, the details are not pursued here. ${ }^{11}$ The system $\chi=F^{1}(\varepsilon)=\varepsilon$ and $\chi=F^{2}(\varepsilon)=2-\varepsilon$, where $\varepsilon$ and $\chi$ are ordinal scalars ranging over all real numbers, has the unique solution $\chi=\varepsilon=1$. Applying the same increasing transformation $T(\varepsilon)=-\lambda^{-\varepsilon}$ used earlier (where $S$ in (10) remains the identity transformation) results in $\chi=F^{1}(T(\varepsilon))=-\lambda^{-\varepsilon}$ and $\chi=F^{2}(T(\varepsilon))=2+\lambda^{-\varepsilon}$. The latter system has no solution since there is no numerical value of $\varepsilon$ for which $\lambda^{-\varepsilon}=-1$.

The general point is that an analysis employing structures containing variables that are only ordinally measured must, of necessity, be grounded in unmeasured relations that lie behind the quantified relations if the latter relations and their properties are to have any significance. The most competent way of providing that grounding is with Approach A. And, although subject to the difficulties described earlier, Approach B respects this grounding too, albeit in a weaker sense. But when the underlying relations and scales on which the ordinal variables are measured are taken to be both unspecified and unknown as in 
Approach C, the grounding in unmeasured relations becomes still weaker and more tenuous, and the analysis requires the imposition of additional restrictions on the transformations of scale that may be used beyond those conditions implied by the ordinality of the measures employed. And the extent of those additional restrictions determines the degree of arbitrariness introduced into the analysis by the presence of the ordinally measured variables.

\section{Preserving Functional Forms}

It turns out that the arbitrariness inherent in Approach C, although coming from a different source, can also, under certain special conditions, extend to Approachs A and B. An important example of this extension in the case of Approach B relates to the production function in what has become known as the efficiency wage model. But before focusing attention on it, it is necessary to consider a more general instance that derives from (1) and (2).

When $x$ is already measured on a ratio scale, as in the case of the production function relating effort to output in the efficiency wage model, the discussion of Sections I and II applies with the obvious alterations. Indeed, since the scale on which $x$, or output, is measured is taken to be fixed, it is only necessary in that discussion to restrict attention to the effect of applying increasing transformations to the measured values of the remaining ordinal variables. To summarize previous argument with respect to Approaches A and B in such a context, observe that (2) may now be rewritten as

$$
x=F(\varepsilon)
$$

where the values of $\varepsilon$, that is, the measured values of $e$, are taken from an appropriate ordinal scale.

Obviously, whenever the scale on which $e$ is measured changes, the measured values of $e$ in $E$ change from $\varepsilon$ to $\varphi$, where, in the notation of the left-hand equation of (3), $\varphi=T(\varepsilon)$, and $T$ is an increasing transformation. But, as before, if (1) is to be preserved as in Approaches A and $\mathrm{B}$, since any particular value of $e$ underlying 
the corresponding measured values $\varepsilon$ and $\varphi$ has not been altered by the transformation of scale, the function value $x=f(e)$ cannot modify. Hence $F$ has to change to offset the modification of scale. Moreover, assuming appropriate inverses exist, any two of $F, G$, and $T$ determines the third. That is, in particular, the reasoning behind equations (4) and (5) applies here except that now $S$ is the identity transformation. Therefore

$$
T=G^{-1} \circ F
$$

and, also as in Section I , $F=G \circ T$ and $G=F \circ T^{-1}$.

It should be noted that (maintaining the perspectives of Approach A or B) if $T$ were the identity transformation, then (15) or either of the above two equations following it would imply that $G=F$. Moreover, with $F$ increasing, $F$ itself is an ordinal scale on which $e$ is measured and $F^{-1}(x)$ provides the measured values of $e=f^{-1}(x)$ on that scale. And this scale and the measured values on it are perfectly valid for use in analysis involving $f$ or $F$.

In the case of Approach $\mathrm{C}$, equation ( $\left.2^{\prime}\right)$ assumes the role of anchor. That, as has been emphasized in the foregoing argument, is the essence of Approach C. Suppose the analytically relevant property to be preserved is the form of $F$ itself. Then changing the scale on which $e$ is measured as in (10) gives (here $S$ is the identity transformation

$$
x=F(T(\varepsilon)) .
$$

Since the left-hand sides of this equation and $\left(2^{\prime}\right)$ are fixed and equal,

$$
F(\varepsilon)=F(T(\varepsilon))
$$


and, as long as $F$ is one-to-one, it follows that

$$
T(\varepsilon)=\varepsilon
$$

Therefore, under these conditions, $T$ is the identity transformation and no changes of scale in reference to $\varepsilon$ can be permitted. This is a rather extreme requirement that severely compromises the usefulness of analysis. Indeed, there is an overwhelming arbitrariness in the selection of $F$ and the scale on which $e$ is measured that cannot be mitigated by even the smallest variation in the latter scale.

Return now to Approach B and consider, for illustrative purposes, the production function of the standard efficiency wage model, namely,

$$
x=F(\varepsilon L)
$$

where $x$ represents output, $\varepsilon$ is effort provided per unit of labor input (assumed to be measured only ordinally), and $L$ is the quantity of labor input employed. In the formulation of (16) the underlying production function $x=f(e, L)$ and the ordinal scale on which $e$ is measured are known without specification and the values of $x$ and $L$ are taken to be measured on known and fixed ratio scales. Furthermore, (16) represents $x=f(e, L)$ given the unspecified scales of measurement.

Of course, the assumption that $x=F(\varepsilon L)$ represents $x=f(e, L)$ would not be permitted under Approach A without the specification of $f$ and explicit relationships (detailed earlier) linking the former to the latter. And such specifications have yet to be provided in the Economics literature. Absent those specifications, the assumption content of the efficiency wage model in terms of the underlying reality to which it is addressed becomes murky, and questions concerning the relevance of the model to actual economic behavior come into play. Moreover, under the assumptions set out above (and one more to be identified below), to preserve the multiplicative structure of (16) across increasing transformations of scale 
applied to $\varepsilon$ requires, as in Approach C, restricting those transformations beyond that necessitated by dint of the ordinality of $\varepsilon$. Of course, a similar conclusion would obtain were (16) to arise with respect to Approach A (where the underlying $f$ and appropriate scales are fully specified) and were it desired to maintain the multiplicative form of the arguments of $F$.

To see what is involved in this last claim, let an alternate scale, $\varphi$, on which effort is measured be given, where

$$
\varphi=T(\varepsilon)
$$

and write the new production function implied by this scale change as in (4):

$$
x=G(\varphi L)
$$

Then combining (16)-(18) gives

$$
F(\varepsilon L)=G(T(\varepsilon) L)
$$

Since $F$ is a production function, it may be assumed to be increasing (the additional assumption mentioned above). Combining this with the previously assumed increasingness of $T$, it follows that $G$ is also increasing. The latter, in turn, implies that $G$ has an inverse so that, from (19),

$$
T(\varepsilon) L=G^{-1}(F(\varepsilon L)) \text {. }
$$

Consider combinations of $\varepsilon$ and $L$ such that 


$$
\varepsilon L=\gamma,
$$

where $\gamma$ is an arbitrary constant. Using (17), (20), and (21),

$$
\varphi=\frac{G^{-1}(F(\varepsilon L))}{\varepsilon L} \varepsilon=\frac{G^{-1}(F(\gamma))}{\gamma} \varepsilon
$$

Since (22) holds for all values of $\varepsilon$ independently of $\gamma$,

$$
\varphi=k \varepsilon
$$

where the constant $k$ is given by

$$
k=\frac{G^{-1}(F(\gamma))}{\gamma} .
$$

Note that, in spite of (24), $k$ must be independent of $\gamma$. For if $k_{1}$ and $k_{2}$ were each associated with a different value of $\gamma$, then from (23), $\varphi=k_{1} \varepsilon$ and $\varphi=k_{2} \varepsilon$. Hence, because $T$ has to remain dependent only on $\varepsilon$ as in (17), $k_{1}=k_{2}$. To show that transformations of the form of (23) are permissible in that they preserve the multiplicative form of $F$ for any constant $k>0$, observe that $\varphi=T(\varepsilon)=k \varepsilon$ and (16) imply

$$
x=F\left(\frac{1}{k} \varphi L\right)
$$

The function $G$ may now be defined as 


$$
G(\varphi L)=F\left(\frac{1}{k} \varphi L\right)
$$

thereby maintaining the required multiplicative form. Thus the above assumptions reduce the admissible transformations of the effort scale to $T(\varepsilon)=k \varepsilon$. This does not mean that effort is actually measured on a ratio scale. But it does demonstrate that, to preserve the underlying structure of the standard efficiency wage production function with output determined by the product of effort and the amount of labor employed, the only ordinal scales that can be invoked as measures of effort are all multiples of each other. Clearly, then, the standard specification of the production function in the efficiency wage model relies on significant assumption content in relation to both an appropriate underlying structure and the choice of scale. That assumption content, moreover, injects an arbitrariness into this particular Approach-B-type analysis similar to that detailed more generally for Approach-C-type analyses above.

\section{Conclusion}

It has been suggested here that, in constructing models to explain observed economic behavior, functions of the form $\chi=F(\varepsilon)$, as in equation (2), where $\varepsilon$ and $\chi$ are ordinally measured variables and where $F$ is assumed to have appropriate properties, can be postulated as long as it is recognized that $F$ actually represents some underlying, unquantified, and possibly unknown relation with corresponding properties. That is, the recognition of the existence of the underlying relation $f$ without its precise specification or knowledge of it, and without precise specification or knowledge of the ordinal scales relating to it (Approaches B and C) is sufficient, if not fully satisfactory, for analysis to proceed. But degrees of arbitrariness and ambiguity, as has been seen, are involved in Approach $\mathrm{C}$ which uses a function like $F$ (rather than the underlying $f$ ) as the anchor of its analyses. And, although not intrinsic to Approach B, a certain arbitrariness and restrictiveness also creeps in here when specific functional forms are imposed, as is the case with the multiplicative-argument specification of the efficiency wage production function. Finally, 
although better than Approaches B and C in principle, Approach A may not be possible to implement and, even when implementation is possible, use of the former may be analytically more tractable. ${ }^{12}$

Moreover, it should be pointed out that the deficiencies of Approaches B and C take on greater significance when explanation is the purpose of the analysis. For explanation requires an elucidation of the reasons for the observed behavior and, for the most part, this means the setting out of the assumptions that are required for the explanation to be relevant and correct. But it is clear in these cases that, because the relationship between $f$ and $F$ is contingent on the ordinal scales invoked, substantial information about $f$ cannot be deduced from $F$ and its properties alone. Thus, to specify only the latter is to leave significant and fundamental assumptions of the model, and hence of the explanation, up in the air. And therefore, what is purported to be an explanation of the observed behavior in question turns out to be, at best, rather incomplete and, at worst, no explanation at all.

\section{References}

Akerlof, George A. (1970) 'The Market for Lemons: Quality Uncertainty and the Market Mechanism,' Quarterly Journal of Economics 84 (3), 488-500.

Akerlof, George A. (1980) 'A Theory of Social Custom of which Unemployment May Be One Consequence,’ Quarterly Journal of Economics 94 (4), 749-775.

Becker, Gary S. and Lewis, H. Gregg (1973) 'On the Interaction between the Quantity and Quality of Children,’ Journal of Political Economy 81 (2, pt.2), S279-S288.

Currie, Martin and Steedman, Ian (1993) ‘Taking Effort Seriously,' Metroeconomica 44 (2), 134-145.

Gintis, Herbert (1995) 'Taking Effort Seriously: A Reply to Currie and Steedman,' Metroeconomica 46 (2), 202-210.

Katzner, Donald W. (1979) Choice and the Quality of Life, Beverly Hills: SAGE.

Katzner, Donald W. (1983) Analysis without Measurement, Cambridge: Cambridge University Press.

Katzner, Donald W. (1996) 'Analysis with Ordinal Measurement,' Employment, Economic Growth and the Tyranny of the Market, v. 2, Philip Arestis, ed. Cheltenham: Elgar, 37-54. Reprinted in Donald W. Katzner (2001) Unmeasured Information and the Methodology of Social Scientific Inquiry, Boston: Kluwer, 197215. 
Katzner, Donald W. (1998) 'The Misuse of Measurement in Economics,' Metroeconomica 49 (1), 1-22. Reprinted in Donald W. Katzner (2001) Unmeasured Information and the Methodology of Social Scientific Inquiry, Boston: Kluwer, 175-195.

Katzner, Donald W. (2001) Unmeasured Information and the Methodology of Social Scientific Inquiry, Boston: Kluwer.

Leslie, Derek (1995) 'On the Proper Use of Ordinal Variables in Labour Market Models,' The Manchester School 63 (2), 196-205.

Skott, Peter (1997a) ‘Measuring Effort,’ Metroeconomica 48 (3), 300-305.

Skott, Peter (1997b) 'On the Proper Use of Ordinal Variables -- A Comment,' The Manchester School 65 (5), 599-601.

Stiglitz, Joseph E. (1987) 'The Causes and Consequences of the Dependence of Quality on Price,' Journal of Economic Literature 25 (1), 1-25.

\section{Footnotes}

1. The authors would like to thank Derek Leslie, Ian Steedman, and especially Douglas Vickers for their help. Thanks are also due to Aarhus Universitets Forskningsfond for partial funding.

2. A ratio scale is a cardinal scale that is unique up to increasing, linear transformations that do not alter the zero value. That is, with respect to cardinal scales in general, the zero on any one scale may be translated into any real number upon application of an appropriate increasing, linear transformation to that scale. But for ratio scales, the zero is fixed and has the same magnitude, namely zero, on all permissibly transformed scales.

3. See Katzner (1983, 2001).

4. See, for example, Katzner (1998: 5,6).

5. Leslie's argument in this regard is not so far from that of Skott. Gintis (1995) also hints at something comparable.

6. The terms "unquantified" and "unmeasured" are used synonomously in this paper as are "quantified" and "measured."

7. It may be noted at this point that some of the analytical problems that will be clarified in what follows turn on the question of whether the model in view and its properites are assumed to be grounded in (2) as opposed to the further-stage-removed relation (1).

8. The fact that utility functions are not usually constructed in this way is irrelevant. For in that case, the variables with respect to which the domain of the utility function is characterized are ratio measured, and the application of increasing transformations to the ordinal scale on which utility is measured have no impact on the demand functions derived.

9. Katzner (1983: Sect. 7.2). 
10. Observe that $S^{-1} \circ F \circ T$ of (10) is different from $G=S \circ F \circ T^{-1}$ which, as indicated earlier (following equation (6)), always preserves the same underlying $f$.

11. General discussion may be found in Katzner (1996: 46-50).

12. Another option when Approach A cannot be implemented or is intractable is to discard all numerical measures of $e$ and $x$ and proceed with $f$ in unquantified form along the analytic lines suggested by Katzner (1983, 2001). 